\title{
MONITORAMENTO EPIDEMIOLÓGICO DA TUBERCULOSE NO ESTADO DA PARAÍBA, BRASIL, 2010 A 2019
}

\author{
EPIDEMIOLOGICAL MONITORING OF TUBERCULOSIS IN THE \\ STATE OF PARAÍBA, BRAZIL, 2010 TO 2019
}

Bueno Callou Bernardo de Oliveira ${ }^{1}$ Pedro Victor Farias do Nascimento ${ }^{2}$ Aline de Paula Rêgo Graciliano Luz ${ }^{3}$ Cláudia Santos Martiniano Sousa ${ }^{4}$ Paula Hino ${ }^{5}$

Tânia Maria Ribeiro Monteiro de Figueiredo ${ }^{6}$

RESUMO: OBJETIVO: Analisar a tendência temporal dos coeficientes de incidência dos casos de tuberculose, tuberculose drogarresistente, tuberculose com Esquema Especial e de micobacterioses não tuberculosas notificados no estado da Paraíba no período de 2010 a 2019. MÉTODOLOGIA: Estudo ecológico utilizando dados do Sistema de Informação de Agravos de Notificação (Sinan) e do Sistema de Tratamentos Especiais da Tuberculose (SITE-TB), aplicando-se a análise de tendência temporal para avaliação da série histórica dos casos através da criação de modelos de regressão polinomial. RESULTADOS: Na série observada, foram notificados 11.335 casos de tuberculose, 139 de tuberculose drogarresistente, 186 de tuberculose com Esquema Especial e 25 de micobacterioses não tuberculosas. Todas as séries temporais apresentaram padrão estável de incidência. CONCLUSÃO: Após análise dos dados, conclui-se que a carga da tuberculose no estado da Paraíba já não apresenta padrão de queda quando avaliada a série histórica recente, mantendo-se temerariamente estável na última década. Para avaliar e monitorar adequadamente a incidência da tuberculose drogarresistente nos

\footnotetext{
${ }^{1}$ Mestre em Saúde Pública pela Universidade Estadual da Paraíba. E-mail: buenocallou@gmail.com.

2 Graduado em Enfermagem pela Universidade Estadual da Paraíba. Aluno do curso de PósGraduação em Saúde Pública da Universidade Estadual da Paraíba. E-mail: pedrofari_14@hotmail.com.

3 Graduada em Enfermagem pela Universidade Estadual da Paraíba. Aluna do curso de PósGraduação em Saúde Pública da Universidade Estadual da Paraíba. E-mail: alinegracluz@gmail.com.

${ }^{4}$ Professora Doutora do curso de Pós-Graduação em Saúde Pública da Universidade Estadual da Paraíba. E-mail: profaclaudiamartiniano@gmail.com.

${ }^{5}$ Professora Doutora da Escola Paulista de Enfermagem da Universidade Federal de São Paulo. Email: paula.hino@unifesp.br.

${ }^{6}$ Professora Doutora do curso de Pós-Graduação em Saúde Pública da Universidade Estadual da Paraíba. E-mail: taniaribeiro@ccbs.uepb.edu.br.
} 
diferentes cenários, faz-se necessário utilizar estimativas da proporção de casos novos de tuberculose com drogarresistência, e não somente proceder a análise dos números absolutos, aplicando-se o mesmo aos casos de tuberculose com Esquema Especial. Apesar da constatação da baixa carga de micobacterioses não tuberculosas no estado, seu monitoramento segue relevante devido ao seu padrão clínico semelhante com o da tuberculose.

Palavras chave: Estudos de Séries Temporais. Micobactérias não Tuberculosas. Monitoramento Epidemiológico. Tuberculose Resistente a Múltiplos Medicamentos.

ABSTRACT: OBJECTIVE: To analyze the time trend of the incidence coefficients of cases of tuberculosis, drug-resistant tuberculosis, tuberculosis with Special Scheme and non-tuberculous mycobacteriosis reported in the state of Paraiba in the period from 2010 to 2019. METHODOLOGY: Ecological study using data from the Information on Notifiable Diseases (Sinan) and the Special Treatment System for Tuberculosis (SITE-TB), applying the time trend analysis to assess the historical series of cases through the creation of polynomial regression models. RESULTS: In the series observed, 11,335 cases of tuberculosis were reported, 139 of drugresistant tuberculosis, 186 of tuberculosis with Special Scheme and 25 of nontuberculous mycobacteriosis. All time series showed a stable incidence pattern. CONCLUSION: After analyzing the data, it can be concluded that the burden of tuberculosis in the state of Paraíba no longer shows a pattern of decline when the recent historical series is evaluated, remaining steadily stable in the last decade. In order to properly assess and monitor the incidence of drug-resistant tuberculosis in different scenarios, it is necessary to use estimates of the proportion of new cases of drug-resistant tuberculosis, and not only proceed to the analysis of absolute numbers, applying the same to cases of tuberculosis with drug resistance. Special Scheme. Despite the finding of a low burden of non-tuberculous mycobacteriosis in the state, its monitoring remains relevant due to its clinical pattern similar to that of tuberculosis.

Keywords: Time Series Studies. Non-Tuberculous Mycobacteria. Epidemiological Monitoring. Tuberculosis Resistant to Multiple Medicines. 


\section{INTRODUÇÃO}

A informação é condição básica para o enfrentamento de qualquer doença/agravo em saúde pública (BRASIL, 2019a). Desde 2006, esforços conjuntos têm sido feitos para atualizar anualmente as estimativas de carga da tuberculose (TB) nos diferentes níveis. Em 2018, foram notificados globalmente 7 milhões de casos novos de TB, mas considerando os problemas de subnotificação, em verdade, cerca de 10 milhões de pessoas desenvolveram a doença no período, correspondendo a um coeficiente de incidência de 132 casos por 100 mil habitantes (WHO, 2019).

De acordo com a Organização Mundial de Saúde (OMS), o coeficiente de incidência da TB apresentou anualmente um declínio médio global de 1,6\% no período de 2000 a 2018. No entanto, preocupa o padrão epidemiológico atual da região das Américas, onde estima-se que a carga da doença esteja aumentando após anos de declínio, sobretudo devido a tendência ascendente registrada no Brasil no período de 2016 a 2018 (WHO, 2019). Embora o coeficiente de incidência da TB no país tenha apresentado queda média anual de $1,7 \%$ de 2007 a 2016, passando de 37,9 casos por 100 mil habitantes em 2007 para 32,4 casos por 100 mil habitantes em 2016 (BRASIL, 2017a), a tendência passou a ser crescente nos anos que se seguiram, suscitando um recrudescimento para 35 casos por 100 mil habitantes no ano de 2019 (BRASIL, 2020).

A análise do perfil epidemiológico, o monitoramento e a avaliação das ações de controle da TB no Brasil são baseadas principalmente nas informações registradas no Sistema de Informação de Agravos de Notificação (Sinan), que é o sistema oficial para o registro das doenças de notificação compulsória no país (BRASIL, 2019a, 2019b; ROCHA, 2020). Os casos de TB que tenham indicação de uso de Esquema Especial de tratamento, após notificados no Sinan, devem ser encerrados de acordo com as suas especificidades por mudança de esquema, falência ou tuberculose drogarresistente (TB DR), prosseguindo-se com sua 
notificação no Sistema de Informação de Tratamentos Especiais de Tuberculose (SITE-TB) (BRASIL, 2019a; BATHOLOMAY, 2019).

Implantado em 2013, o SITE-TB surgiu da necessidade de monitorar rotineiramente as pessoas com TB DR no Brasil, contudo além dos casos que envolvem drogarresistência, também são notificados no sistema os casos de TB sensível com indicação de uso Esquema Especial de tratamento (TB com Esquema Especial) e casos de micobacterioses não tuberculosas (MNT), identificadas por diagnóstico diferencial de TB (BATHOLOMAY, 2019).

Os Sistemas de Informação em Saúde possibilitam a identificação do progresso, dos problemas e das necessidades que envolvem os diferentes cenários, e assim se constituem como base do processo informação-decisão-ação (BRASIL, 2019a; MUTALE, 2013; THOMAZ, 2015).

Dessa forma, considerando que a informação é condição básica para o processo de tomada de decisão, face ao aporte de dados fornecidos pelo Sinan e pelo SITE-TB, e tendo em vista a necessidade de priorizar pesquisas periódicas para mensurar a carga da TB nos diferentes níveis, monitorando todas as suas condições, este estudo teve como objetivo analisar a tendência temporal dos coeficientes de incidência dos casos de tuberculose, tuberculose drogarresistente, tuberculose com Esquema Especial e de micobacterioses não tuberculosas notificados no estado da Paraíba no período de 2010 a 2019.

\section{MÉTODOS}

Este é um estudo ecológico utilizando dados do Sinan e do SITE-TB para análise de tendência temporal dos coeficientes de incidência dos casos novos de TB, TB DR, TB com Esquema Especial e de MNT notificados no estado da Paraíba no período de 2010 a 2019.

O cenário de estudo é um estado do Nordeste brasileiro com uma população estimada de aproximadamente quatro milhões de habitantes em 2019, com área 
territorial de 56.468,435 km², organizado em 223 municípios, e com um Índice de Desenvolvimento Humano (IDH) de 0,658 em 2010 (IBGE, 2019a).

Foram incluídos no estudo os casos de TB em que o tipo de entrada foi registrado no Sinan como caso novo, não sabe ou pós-óbito, e os casos de TB DR, TB com Esquema Especial e de MNT em que o tipo de entrada foi registrado no SITETB como caso novo, mudança de esquema ou outros. Os casos com a situação de encerramento registrada como mudança de diagnóstico, e os casos duplicados quando verificadas as variáveis nome, data de nascimento e nome da mãe. foram excluídos da análise.

Para as estimativas populacionais utilizadas no cálculo do coeficiente de incidência dos casos de TB e de MNT, foram utilizados os dados do Instituto Brasileiro de Geografia e Estatística (IBGE) (IBGE, 2019b). Os coeficientes de incidência dos casos de TB DR e de TB com Esquema Especial foram calculados obtendo-se a razão dos casos novos de TB DR e de TB com Esquema Especial pelos casos novos de TB.

Com a finalidade de caracterizar a amostra, inicialmente foi realizada a análise descritiva dos casos. Em seguida, a análise de tendência temporal foi utilizada para avaliação dos dados, com a criação de modelos de regressão polinomial, cujo objetivo principal foi encontrar a curva que melhor se adequasse aos dados e descrevesse a relação entre a incidência (variável dependente) e o tempo/ano estudado (variável independente), e dessa forma identificar se a incidência apresentou tendência estável, crescente ou decrescente durante o período de observação. Os seguintes modelos de regressão polinomial foram testados: a) linear; b) quadrático; c) cúbico.

A escolha do melhor modelo foi determinada pela análise do gráfico de dispersão com base no coeficiente de determinação $\left(R^{2}\right)$, para o qual valores mais próximos de 1 indicam melhor qualidade de ajuste e análise de resíduos (suposição de homoscedasticidade verdadeira). Quando dois modelos foram semelhantes do ponto de vista estatístico, o modelo mais simples (ordem inferior) foi escolhido para evitar uma correlação serial entre períodos (anos) (MONTGOMERY, 2015). O modelo cúbico foi o que melhor se ajustou aos dados. 
Este estudo fez parte do projeto multicêntrico intitulado "A tuberculose drogarresistente no estado da Paraíba: um desafio para saúde pública", o qual foi aprovado pelo Comitê de Ética em 17/12/2020, sob o Parecer $n^{\circ}$ 24127319.3.0000.5187.

\section{RESULTADOS}

Como demonstrado na tabela 1, no período de 2010 a 2019 foram notificados no estado da Paraíba 11.335 casos novos de TB, 139 casos novos de TB DR, 186 casos novos de TB com Esquema Especial e 25 casos novos de MNT.

Tabela 1 - Descrição da série histórica dos casos de tuberculose, tuberculose drogarresistente, tuberculose com Esquema Especial e de micobacterioses não tuberculosas. Paraíba, 2010 a 2019.

\begin{tabular}{ccccc}
\hline ANO & TB $^{(\mathbf{a})}$ & MNT $^{(\mathbf{b})}$ & TB DR $^{(\mathbf{c})}$ & TB com Esquema Especial $^{(\mathbf{a})}$ \\
\hline 2010 & 1.030 & 0 & 5 & 0 \\
2011 & 1.081 & 0 & 5 & 8 \\
2012 & 1.137 & 0 & 11 & 18 \\
2013 & 1.142 & 4 & 8 & 26 \\
2014 & 1.049 & 3 & 13 & 17 \\
2015 & 1.045 & 8 & 30 & 26 \\
2016 & 1.149 & 0 & 17 & 23 \\
2017 & 1.098 & 1 & 21 & 14 \\
2018 & 1.302 & 5 & 14 & 36 \\
2019 & 1.302 & 4 & 15 & 18 \\
\hline Total & 11.335 & 25 & 139 &
\end{tabular}
a) Tuberculose;
b) Micobacterioses não tuberculosas;
c) Tuberculose drogarresistente;
d) Tuberculose sensível com indicação de uso de Esquema Especial de tratamento;

Na Tabela 2 observa-se que o menor coeficiente de incidência dos casos de TB foi registrado em 2015 (26,31 casos por 100 mil habitantes), e o maior coeficiente 
foi registrado em 2018 (32,58 casos por 100 mil habitantes). Em 2010, 2011, 2012 e 2016 não foram registrados casos de MNT no estado, e o maior coeficiente de incidência foi registrado em 2015 (0,20 casos por 100 mil habitantes).

Tabela 2 - Análise de tendência dos coeficientes de incidência dos casos de tuberculose e de micobacterioses não tuberculosas. Paraíba, 2010 a 2019.

\section{Coeficiente de incidência x 100 mil habitantes}

\begin{tabular}{ccc}
\hline ANO & TB $^{(\mathbf{a})}$ & $\mathbf{M N T}^{\mathbf{( b )}}$ \\
\hline 2010 & 27,34 & - \\
2011 & 28,51 & - \\
2012 & 29,80 & - \\
2013 & 29,17 & 0,10 \\
2014 & 26,60 & 0,08 \\
2015 & 26,31 & 0,20 \\
2016 & 28,73 & - \\
2017 & 27,28 & 0,02 \\
2018 & 32,58 & 0,13 \\
2019 & 32,40 & 0,10 \\
\hline Tendência $^{2(c)}$ & Estável & Estável \\
\hline $\mathrm{R}^{(\mathbf{c})}$ & 0,679 & 0,294 \\
\hline p-valor & 0,063 & 0,294
\end{tabular}
a) Tuberculose;
b) Micobacterioses não tuberculosas;
c) Coeficiente de determinação com base no modelo cúbico;
d) Regressão polinomial. 
$\mathrm{Na}$ análise de tendência temporal constatou-se que os coeficientes de incidência dos casos de TB $\left(R^{2}=0,679 ; p=0,063\right)$, e de MNT $\left(R^{2}=0,294 ; p=\right.$ $0,524)$ apresentaram padrões estáveis no período de observação, com discretas variações anuais.

Na Tabela 3 observa-se que o menor coeficiente de incidência dos casos de TB DR foi registrado em 2011 (0,47 casos por 100 casos de TB), e o maior coeficiente foi registrado em 2015 (2,96 casos por 100 casos de TB). Em 2010 não foram registrados casos de TB com Esquema Especial no estado, e o maior coeficiente de incidência foi registrado em 2018 (2,82 casos por 100 casos de TB).

Tabela 3 - Análise de tendência dos coeficientes de incidência dos casos de tuberculose drogarresistente e de tuberculose com Esquema Especial. Paraíba, 2010 a 2019.

\begin{tabular}{|c|c|c|}
\hline \multicolumn{3}{|c|}{ Coeficiente de incidência $\times 100$ casos novos de TB ${ }^{(a)}$} \\
\hline ANO & TB DR ${ }^{(\mathbf{b})}$ & TM com Esquema Especial ${ }^{(c)}$ \\
\hline 2010 & 0,49 & - \\
\hline 2011 & 0,47 & 0,75 \\
\hline 2012 & 0,99 & 1,61 \\
\hline 2013 & 0,71 & 2,32 \\
\hline 2014 & 1,28 & 1,67 \\
\hline 2015 & 2,96 & 2,56 \\
\hline 2016 & 1,54 & 2,08 \\
\hline 2017 & 1,99 & 1,23 \\
\hline 2018 & 1,13 & 2,82 \\
\hline 2019 & 1,25 & 1,33 \\
\hline Tendência & Estável & Estável \\
\hline $\mathrm{R}^{2(\mathrm{~d})}$ & 0,583 & 0,666 \\
\hline$p$-valor ${ }^{(e)}$ & 0,132 & 0,071 \\
\hline \multicolumn{3}{|l|}{ a) Tuberculose; } \\
\hline \multicolumn{3}{|c|}{ b) Tuberculose drogarresistente; } \\
\hline \multicolumn{3}{|c|}{ c) Tuberculose sensível com indicação de uso de Esquema Especial de tratamento; } \\
\hline \multicolumn{3}{|c|}{ d) Coeficiente de determinação com base no modelo cúbico; } \\
\hline e) Regressão polinor & & \\
\hline
\end{tabular}


$\mathrm{Na}$ análise de tendência temporal constatou-se que os coeficientes de incidência dos casos de TB DR $\left(R^{2}=0,583 ; p=0,132\right)$, e de TB com Esquema Especial $\left(R^{2}=0,666 ; p=0,071\right)$, também apresentaram padrões estáveis no período de observação, com discretas variações anuais.

\section{DISCUSSÃO}

No estudo realizado por Melo, Barros e Donalisio (2020), sobre a tendência temporal dos coeficientes de incidência da TB nas regiões e estados brasileiros, com análise da série histórica de 2001 a 2017, o estado da Paraíba apresentou um percentual de $1,6 \%$ de queda média anual no coeficiente de incidência da doença. Contrapondo tal resultado, este estudo constatou que a tendência temporal da incidência da TB no estado da Paraíba já não apresenta variações anuais decrescentes quando analisada a série histórica recente.

Destaca-se ainda, que apesar da estabilidade observada na série temporal, preocupa o fato dos maiores coeficientes terem sido registrados nos anos de 2018 e de 2019, corroborando assim com os resultados das análises nacionais divulgadas no Boletim Epidemiológico de Tuberculose (2020), as quais indicam a possibilidade de tendência crescente da incidência da TB no Brasil nos últimos anos.

Para avaliar se a implantação Xpert MTB/RIF aumentaria a taxa de notificação da TB no Brasil, um ensaio clínico randomizado foi realizado em duas capitais brasileiras no ano de 2012, entretanto nenhum aumento nas taxas gerais de notificação foi observado, possivelmente devido aos altos índices de tratamento empírico da doença no país (DUROVNI, 2014). A Rede de Teste Rápido para Tuberculose (RTR-TB) foi implantada em todas as Unidades da Federação no ano de 2014, com 167 equipamentos distribuídos em 92 municípios, sendo expandida nos anos de 2017 e 2018, com a aquisição de mais 70 equipamentos, ampliando para 128 municípios com oferta do Xpert MTB/RIF na rede pública de saúde (BRASIL, 2015, 2018). Desta forma, novos estudos são necessários para avaliar se 
a implantação, ampliação e consolidação da RTR-TB no estado da Paraíba relaciona-se com o aumento dos casos de TB registrado nos últimos anos.

Observa-se neste estudo que o coeficiente de incidência das MNT se manteve sempre abaixo de 1 caso por 100 mil habitantes nos anos observados, resultado que demonstra a baixa carga deste agravo no estado da Paraíba. $O$ diagnóstico das micobacterioses pulmonares é complexo e exige uma combinação de achados clínicos, radiológicos e microbiológicos segundo critérios estabelecidos pela American Thoracic Society/Infectious Diseases Society of America (ATS/IDSA) (CARNEIRO, 2018; MARQUES, 2019). Há poucos estudos no Brasil sobre doença pulmonar por MNT, e estes em sua maioria são estudos que restritos a certas localidades, tornando difícil estimar sua prevalência no país (DE MELO, 2013).

Apesar da constatação da baixa carga de MNT no estado, seu monitoramento segue relevante devido ao seu padrão clínico semelhante com o da TB. A apresentação clínica mais frequente das MNT é a pulmonar, e os sintomas incluem tosse crônica produtiva, dispnéia, hemoptise, febre e perda de peso, sendo assim plausível que muitos desses casos sejam tratados como TB, resultando muitas vezes em situações de resposta inadequada ao tratamento. Particularmente na população indígena, o diagnóstico da TB pulmonar não deve ser baseado na radiografia de tórax, pois há maior ocorrência de infecção por MNT nesta população (BRASIL, 2019a).

Para avaliar e monitorar a incidência da TB DR nos diferentes cenários, a OMS utiliza estimativas da proporção de casos novos de TB com drogarresistência. De acordo com a organização, a região das Américas, igualmente a região Africana, registrou a menor proporção de casos novos de TB com drogarresistência em 2018, com a notificação de 2,5 (1.5 - 3.8 IC) casos novos para cada 100 casos novos de TB notificados (WHO, 2019).

No Brasil, o Ministério da Saúde não divulga análises da proporção de casos novos de TB com drogarresistência. Até 2017 o número absoluto de casos novos de TB DR notificados no país era publicado anualmente no Boletim Epidemiológico de Tuberculose, no entanto com a publicação neste mesmo ano do Plano Nacional pelo Fim da Tuberculose como Problema de Saúde Pública, retirando o "número de casos de tuberculose drogarresistente" da lista de indicadores prioritários para o 
monitoramento da TB, todas as publicações dos Boletins Epidemiológicos de Tuberculose que se seguiram não mais abordaram as estimativas de carga da TB DR (BRASIL, 2017a, 2017b).

Em 2019 foi publicado o Manual de Recomendações para o controle da Tuberculose no Brasil, o qual trouxe novamente o "número de casos novos de tuberculose drogarresistente" como indicador prioritário. O manual também trouxe o quantitativo de casos novos de drogarresistência notificados no país em 2017, ano em que foram registrados no SITE-TB 246 casos de monorresistência, 80 casos de polirresistência, 713 de multidrogarresistência (MDR) / resistência à rifampicina (RR) e 2 casos de resistência extensiva (XDR).

Observa-se neste estudo que o estado da Paraíba apresenta padrão estável na incidência da TB DR, com baixas proporções anuais; contudo, é preciso frisar que ainda se faz necessário avançar no diagnóstico e notificação desses casos. Nos últimos anos, apenas $63 \%$ dos casos de TB DR estimados para o Brasil foram detectados (BARTHOLOMAY, 2020). Em 2017 foram notificados 1.100 casos de TB MDR no país, correspondendo apenas a $45,8 \%$ dos 2.400 casos estimados pela OMS (TOURINHO, 2020).

Não foram encontrados estudos sobre a carga da TB com Esquema Especial no Brasil, todavia percebe-se neste estudo que a incidência da TB com Esquema Especial foi superior a incidência da TB DR em quase todos os anos observados, demonstrando a necessidade de fomentar pesquisas para melhor compreensão de sua magnitude, distribuição, e dos seus fatores associados.

Os resultados deste estudo devem ser considerados sob algumas condições limitantes, sobretudo no que diz respeito aos problemas de subnotificação, duplicação e incompletude de dados nos sistemas de informação (BARTHOLOMAY, 2020; ROCHA, 2020). Para diminuir erros de notificação, falhas no acompanhamento dos casos e subnotificações, é primordial haver convergência das informações entre os níveis de gestão, com implementação de uma rotina permanente de qualificação dos dados (BARTHOLOMAY, 2020; BRASIL, 2019b; ROCHA, 2020).

Também se faz necessário considerar como fragilidade as limitações metodológicas inerentes aos estudos ecológicos, tendo em vista que nesse desenho metodológico as variáveis extrínsecas, as quais funcionam como fatores de 
confundimento, não são consideradas. Entretanto, é preciso ressaltar que a utilização desse tipo de estudo possui vantagens importantes, como ampla cobertura populacional, baixo custo e menor tempo para execução, características que viabilizam o desenvolvimento de pesquisas periódicas para mensurar a carga da TB nos diferentes níveis, com o devido monitoramento de todas as suas condições.

\section{CONCLUSÃO}

Após análise dos dados, conclui-se que a carga da TB no estado da Paraíba já não apresenta um padrão de queda quando avaliada a série histórica recente, mantendo-se temerariamente estável na última década, tornando distante o alcance das metas propostas pela OMS para acabar com a epidemia da TB até 2035 (WHO, 2013).

Para avaliar e monitorar adequadamente a incidência da TB DR nos diferentes cenários faz-se necessário seguir os padrões da OMS, utilizando estimativas da proporção de casos novos de TB com drogarresistência, e não somente proceder a análise dos números absolutos, possibilitando assim a comparação dos dados locais com os dados estimados pela organização. Este mesmo tipo de análise deve ser aplicado aos casos de tuberculose com Esquema Especial, uma condição que carece de pesquisas para melhor compreensão de sua magnitude, distribuição, e dos seus fatores associados.

Apesar da constatação da baixa carga de MNT no estado, seu monitoramento segue relevante devido ao seu padrão clínico semelhante com o da TB, e dessa maneira os casos precisam ser devidamente registrados e acompanhados no SITETB. 


\section{REFERÊNCIAS BIBLIOGRÁFICAS}

BARTHOLOMAY, P. et al. Sistema de Informação de Tratamentos Especiais de Tuberculose (SITE-TB): histórico, descrição e perspectivas. Epidemiol. Serv. Saude, Brasília, 28(2):e2018158, 2019. Disponível em: https://www.scielo.br/pdf/ress/v28n2/2237-9622-ress- 2802-e2018158.pdf.

BARTHOLOMAY, P. et al. Lacunas na vigilância da tuberculose drogarresistente: relacionando sistemas de informação do Brasil. Cad. Saúde Pública 2020; 36(4):e00082219. Disponível em: http://cadernos.ensp.fiocruz.br/static/arquivo/1678-4464-csp-36-05- e00082219.pdf.

BRASIL. Ministério da Saúde. Secretaria de Vigilância em Saúde. Rede de Teste Rápido para Tuberculose no Brasil - Primeiro ano de implantação. Brasília: Ministério da Saúde; 2015. Disponível em: http://www.saude.gov.br/images/pdf/2016/janeiro/15/rtr-tb-15jan16- isbn-web.pdf.

BRASIL. Ministério da Saúde. Secretaria de Vigilância em Saúde. Boletim epidemiológico. Brasília: Ministério da Saúde, v. 48, n. 8, 2017a. Disponível em: https://www.saude.gov.br/images/pdf/2017/marco/23/2017-V-48-N-8-Indicadores-priorit--riospara-o-monitoramento-do-Plano-Nacional-pelo-Fim-da-Tuberculose-como-Problema-de-Sa--deP--blica-no-Brasil.pdf.

BRASIL. Ministério da Saúde. Secretaria de Vigilância em Saúde. Departamento de Vigilância das Doenças Transmissíveis. Brasil Livre da Tuberculose: Plano Nacional pelo Fim da Tuberculose como Problema de Saúde Pública. Ministério da Saúde, Secretaria de Vigilância em Saúde, Departamento de Vigilância das Doenças Transmissíveis. - Brasília: Ministério da Saúde, 2017b. Disponível em: https://bvsms.saude.gov.br/bvs/publicacoes/brasil_livre_tuberculose_plano_nacional.pdf.

BRASIL. Ministério da Saúde. Secretaria de Vigilância em Saúde. Departamento de Vigilância Epidemiológica. Boletim Epidemiológico. Brasília: Ministério da Saúde. v.49, n. 11, 2018. Disponível em: http://www.saude.gov.br/images/pdf/2018/marco/26/2018-009.pdf.

BRASIL. Ministério da Saúde. Secretaria de Vigilância em Saúde. Departamento de Vigilância das Doenças Transmissíveis. Manual de recomendações para o controle da tuberculose no Brasil [Internet]. 2. ed. Brasília: Ministério da Saúde; 2019a. 364 p.

Disponível em:

saude.gov.br/images/pdf/2019/marco/28/manualrecomendacoes.pdf

http://portalarquivos2.

BRASIL. Ministério da Saúde. Sistema de Informação de Agravos de Notificação. Vigilância epidemiológica da tuberculose: Análise de indicadores operacionais e epidemiológicos a partir da base de dados do Sinan versão 5.0. Brasília; 2019b. 53 p. Disponível em: https://portalsinan.saude.gov.br/images/documentos/Agravos/Tuberculose/Caderno_de_Anali se_2019.pdf.

BRASIL. Ministério da Saúde. Secretaria de Vigilância em Saúde. Departamento de Vigilância Epidemiológica. Boletim Epidemiológico. Brasília: Ministério da Saúde. Número Especial, 2020. Disponível em: https://www.saude.gov.br/images/pdf/2020/marco/24/Boletim-tuberculose2020-marcas--1-.pdf.

CARNEIRO, M. S. et al. Doença pulmonar por micobactérias não tuberculosas em uma região de alta incidência de tuberculose no Brasil. J Bras Pneumol. 2018;44(2):106-111 Disponível em: https://www.scielo.br/scielo.php?pid=S1806- 37132018000200106\&script=sci_abstract\&tlng=pt. 
DE MELLO, K. G. et al. Clinical and therapeutic features of pulmonary nontuberculous mycobacterial disease, Brazil, 1993-2011. Emerg Infect Dis. 2013;19(3):393-9 Disponível em: https://www.ncbi.nlm.nih.gov/pmc/articles/PMC3647650/pdf/12-0735.pdf.

DUROVNI, B. et al. Impact of replacing smear microscopy with Xpert MTB/RIF for diagnosing tuberculosis in Brazil: a steppedwedge cluster-randomized trial. PLoS Med, 2014 Dec;11(12):e1001766. Disponível em: https://journals.plos.org/plosmedicine/article/file?id=10.1371/journal.pmed.1001766\&type=printab le.

IBGE. Cidades e estados. Instituto Brasileiro de Geografia e Estatística; 2019a. Disponível em: https://www.ibge.gov.br/cidades-e-estados/pb.html.

IBGE. Estimativas da população. Instituto Brasileiro de Geografia e Estatística; 2019b. Disponível em: https://www.ibge.gov.br/estatisticas/sociais/populacao/9103-estimativas-depopulacao.html?=\&t=downloads.

MARQUES, L. R. M.; FERRAZOLI, L.; CHIMARA, E. Micobacterioses pulmonares: diagnóstico presuntivo pelos critérios microbiológicos internacionais adotados no estado de São Paulo, Brasil, 2011-2014. J Bras Pneumol. 2019;45(2):e20180278. Disponível em: https://www.scielo.br/pdf/jbpneu/v45n2/pt_1806-3713-jbpneu-45-02-e20180278.pdf.

MELO, M. C.; BARROS, H.; DONALISIO, M. R. Temporal trend of tuberculosis in Brazil. Cad. Saúde Pública, 2020; 36(6):e00081319. Disponível em: https://www.scielo.br/pdf/csp/v36n6/1678-4464-csp-36-06-e00081319.pdf.

MONTGOMERY, D. C.; JENNINGS, C. L.; KULAHCI, M. Introduction to Time Series Analysis and Forecasting. 2th Ed. Hoboken, NJ, John Wiley \& Sons, 2015, 672p.

MUTALE, W. et al. Improving health information systems for decision making across five subSaharan African countries: Implementation strategies from the African Health Initiative. BMC Health Serv Res 13, S9 (2013). Disponivel em: https://doi.org/10.1186/1472- 6963-13-S2-S9.

ROCHA, M. S. et al. Sistema de Informação de Agravos de Notificação (Sinan): principais características da notificação e da análise de dados relacionada à tuberculose. Epidemiol. Serv. Saude, Brasília, 29(1):e2019017, 2020. Disponível em: https://www.scielo.br/pdf/ress/v29n1/2237-9622-ress-29-01-e2019017.pdf.

THOMAZ, E. B. A. F. et al. Conceitos e ferramentas da epidemiologia. São Luís: EDUFMA, 2015. Disponível em: https://ares.unasus.gov.br/acervo/html/ARES/7462/1/Livro\%202\%20$\% 20$ Conceitos $\% 20$ e $\% 20$ ferramentas $\% 20$ da $\% 20$ epidemiologia.pdf.

TOURINHO, B. D. et al. Avaliação do Sistema de Vigilância da Tuberculose Drogarresistente, Brasil, 2013-2017. Epidemiol. Serv. Saude, Brasília, 29(1):e2019190, 2020. Disponível em: https://www.scielo.br/pdf/ress/v29n1/2237-9622-ress-29-01- e2019190.pdf.

WHO. Global strategy and targets for tuberculosis prevention, care and control after 2015 [Internet]. Geneva: World Health Organization; 2013 [cited 2017 May 26]. 2 p. Disponível em: https://www.who.int/tb/strategy/End_TB_Strategy.pdf.

WHO. Global Tuberculosis Report 2019. Geneva: World Health Organization; 2019. Licence: CC BY-NC-SA 3.0 IGO. Disponível em: https://www.who.int/tb/publications/global_report/en/. 